\title{
CRYSTAL GROWTH AND DISSOLUTION OF CALCIUM PHOSPHATES: A KINETICS AND SURFACE ENERGY APPROACH
}

\author{
GEORGE H. NANCOLLAS and WENJU WU \\ Chemistry Department, State University of New York at Buffalo \\ Buffalo, N. Y. 14260-3000 USA
}

\begin{abstract}
Constant composition methods have been used to investigate the mechanisms of crystal growth and dissolution of calcium phosphate minerals. Interfacial tensions between water and each of these surfaces were calculated from measured contact angles using surface tension component theory. The interfacial tension values, $-4.2,4.3,10.4$ and $18.5 \mathrm{mJm}^{-2}$ for dicalcium phosphate dihydrate (DCPD), octacalcium phosphate (OCP), hydroxyapatite (HAP) and fluorapatite (FAP), respectively, compare well with those calculated from dissolution kinetics experiments and provide information concerning the growth and dissolution mechanisms. The much smaller interfacial tensions of OCP and DCPD in contact with water compared with those of HAP and FAP support the observation that the crystallization of the latter phases was often preceded by the formation OCP and DCPD and that both OCP and DCPD could serve as precursors to apatite growth. In addition, the ability of a surface to nucleate mineral phases is closely related to the magnitude of the interfacial energies which are also important in understanding stability of calcium phosphate colloidal dispersions.
\end{abstract}

\section{INTRODUCTION}

The interfacial free energy is of the utmost importance in determining the thermodynamics and kinetics of crystal growth and dissolution of calcium phosphates in aqueous solution. For many sparingly soluble minerals, there is a considerable amount of data on crystallization and dissolution. Unfortunately, however, it is difficult to use them for interpreting interfacial energies since they are subject to numerous uncertainties ${ }^{1}$. Thus, the reported values of the interfacial tension obtained for the same solid surface in contact with water may vary markedly not only by using different techniques and models but even with 
the same method. For example, gypsum-water interfacial tensions, obtained from solubility studies, were 26, 370, and $1050 \mathrm{~mJ} \mathrm{~m}^{-2}$ from Stumm ${ }^{2}$, Dundon and Mark and Jones ${ }^{4}$, respectively, while the values derived from homogeneous nucleation kinetic data were 8 and $330 \mathrm{~mJ} \mathrm{~m}^{-2}$ by Ratinov and Todes ${ }^{5}$, and Nielsen ${ }^{6}$, respectively. Values determined from the kinetics and solubility studies on metal oxides, carbonates, sulfates, fluorides, and phosphates were significantly higher than those from contact angle measurements ${ }^{1,2}$. It is therefore not possible to use these values to compare crystallization thermodynamic data with other physicochemical phenomenan such as solubility, adsorption at solid-liquid interfaces, and the stability of crystal particles in aqueous systems.

Conventional methods for studying interfacial free energies based on crystallization and dissolution kinetics are problematic because of the variation, with time, of the solution undersaturation/supersaturation and the very small concentration changes during the free drift to equilibrium. Using free drift methods, although it would appear that kinetic data for crystal growth as a function of supersaturation can be obtained from a single experiment, large errors are often involved in the determination of the rates, especially at low supersaturation. The constant composition technique overcomes these problems and offers an unique opportunity to minimize the variables for investigating interfacial free energy, especially at very low driving forces. In present study, the interfacial tensions at calcium phosphate minerals/water interfaces were characterized using both crystallization/dissolution kinetics and thin layer wicking approaches. Their role in crystallization and dissolution kinetics is also discussed.

\section{EXPERIMENTAI PROCEDURES}

\section{Interfacial tension determined from kinetics:}

The rate, $\mathrm{J}$, of crystallization and dissolution from solution is typically expressed in terms of equation (1):

$$
J=A \exp \left[f\left(T, S, \gamma_{s \ell}\right)\right]
$$

where $\mathrm{A}$ is a pre-exponential factor, $\mathrm{T}$, the temperature and $\gamma_{s \ell}$ is the interfacial tension. $f, \mathrm{a}$ function of $T, S$ and $\gamma_{s \ell}$, will depend on the mechanisms of crystal growth and dissolution 
and has been the subject of numerous studies ${ }^{1}$. Equation (1) can also be rewritten in the form

$$
\ln J=\ln \mathrm{A}+f\left(T, S, \gamma_{s \ell}\right)
$$

Therefore, at constant temperature (T), the interfacial tension, $\gamma_{s c}$, can estimated from a plot of $\operatorname{lnJ}$ against $S$, and numerous attempts have been made to obtain crystal/solution interfacial tensions using this approach ${ }^{1}$. It is most important to maintain constancy of temperature and reaction solution composition when $\gamma_{s \ell}$ is to be determined from crystallization or dissolution kinetics. In the present study, the constant composition (CC) method was used to study crystallization and dissolution kinetics. Using this approach, the titrant solutions containing the lattice ions are simultaneously added to the reaction solutions to compensate for their removal due to crystal growth. Detailed experimental procedures can be found in previous Nancollas publications.

Interfacial tension determined from contact angle measurements

The surface tension components and interfacial tensions between solids and liquids were determined by direct contact angle measurements (sessile drop) for smooth flat surfaces and thin layer wicking for powders. Detailed procedures can be found in previous publications ${ }^{1}$.

\section{RESULTS AND DISCUSSION}

Surface tension components and interfacial tension: Surface tension components were determined at $20^{\circ} \mathrm{C}$ on a number of calcium phosphates that are not available in large, single crystals suitable for direct contact angle measurements (Table 1) using thin layer wicking method. These calcium phosphates include crystals of dicalcium phosphate dihydrate $\left[\mathrm{CaHPO}_{4} \bullet 2 \mathrm{H}_{2} \mathrm{O}, \mathrm{DCPD}\right]$, octacalcium phosphate $\left[\mathrm{Ca}_{8} \mathrm{H}_{2}\left(\mathrm{PO}_{4}\right)_{6} \bullet 5 \mathrm{H}_{2} \mathrm{O}, \mathrm{OCP}\right.$, hydroxyapatite $\left[\mathrm{Ca}_{10}\left(\mathrm{PO}_{4}\right)_{6}(\mathrm{OH})_{2}, \mathrm{HAP}\right]$, and fluorapatite $\left[\mathrm{Ca}_{10}\left(\mathrm{PO}_{4}\right)_{6} \mathrm{~F}_{2}, \mathrm{FAP}\right]$. From the surface tension components, the interfacial tension of each phase in contact with water was calculated ${ }^{1}$. The interfacial tensions were also estimated from kinetics studies at $37^{\circ} \mathrm{C}$ and $0.15 \mathrm{~mol} \mathrm{~L}^{-1}$ ionic strength and are summarized in Table 1 . 
TABLE 1 Surface tension components $\left(\mathrm{mJ} \mathrm{m}^{-2}\right)$ and interfacial tension $\left(\mathrm{mJ} \mathrm{m}^{-2}\right)$ between crystals and solutions for calcium phosphate minerals.

\begin{tabular}{|c|c|c|c|c|c|c|c|}
\hline Mineral & $\gamma_{S}^{L W}$ & $\gamma_{S}^{+}$ & $\gamma_{S}^{-}$ & $\gamma_{S L}^{C}$ & $\gamma_{S L}^{K D}$ & $\gamma_{S L}^{K G}$ & $\log S_{\infty}$ \\
\hline DCPD & 26.4 & 1.55 & 31.7 & -4.2 & 0.4 & 0.4 & -2.22 \\
\hline OCP & 21.6 & 2.16 & 19.7 & 4.3 & 2.9 & 7.1 & -6.16 \\
\hline HAP & 36.2 & 0.92 & 16.0 & 10.0 & 9.3 & 17.1 & -6.44 \\
\hline FAP & 32.4 & 0.64 & 8.96 & 18.5 & 15.0 & 30.0 & -6.67 \\
\hline
\end{tabular}

$\gamma^{\mathrm{LW}}$ is the Lifshitz-van der Waals (LW) surface tension component (apolar); $\gamma^{+}$the electron-acceptor (Lewis acid) parameter; and $\gamma^{-}$the electron donor (Lewis base) parameter. $\mathrm{C}, \mathrm{KD}, \mathrm{KG}, \mathrm{S}_{\infty}$ refer to the contact angle, kinetics of dissolution, kinetics of crystallization and solubility, respectively.

In table 1, it can be seen that the interfacial tension values between the solution and each of the mineral phases, calculated from the kinetics of crystallization and dissolution, are of the same order of magnitude as those obtained from contact angle measurements.

Crystal growth and dissolution: Despite the fact that DCPD is the most soluble among the calcium phosphate minerals, it forms under many physiological, geochemical, and laboratory conditions. Although the basic mechanisms which allow preferential formation of DCPD have been discussed extensively, they remain poorly understood. Since this is a kinetic phenomenon, it may be explained in terms of the low interfacial tension values. At small thermodynamic driving forces for crystallization or dissolution, low interfacial tensions increase the tendency for the initial precipitation or dissolution, of DCPD or OCP amongst the calcium phosphates, as compared with HAP or FAP which have relatively high interfacial tensions. However, surfaces with the lowest interfacial tensions also indicate a thermodynamic instability, and this may explain why DCPD and OCP have been so frequently implicated as possible precursors to the formation of apatites, especially in biological mineralization reactions (tooth, bone, calculus, and renal stone formation).

Colloid and surface properties of calcium phosphates: The surface or interfacial properties, along with the electrostatic interactions control flocculation, adsorption and 
wetting. It is commonly assumed that water wets the surface of a hydrophilic solid and does not wet the surface of hydrophobic solid. This nomenclature is purely desciptive and uninformative about the causes of the particlular behavior described. There is also no indication of a scale of magnitude in this nomenclature, that is, there are surfaces which are more hydrophobic than others, yet the difference between two such surfaces cannot be quantified solely using the terms hydrophobic or hydrophilic. Thermodynamically, particles with $\gamma_{s}^{-}>28 \mathrm{mJm}^{-2}$ may be considered to be hydrophilic while particles with $\gamma_{s}^{-}<28 \mathrm{mJm}^{-2}$ are hydrophobic ${ }^{13}$. The hydrophilicity or hydrophobicity of calcium phosphate biomineral surfaces vary widely; for example, DCPD is hydrophilic and relatively quite soluble, while HAP is more hydrophobic and much less soluble. Although the atomic positions in the OCP crystal structure are almost superimposable on those for HAP, the composition and atomic positions on the surfaces are probably quite different. Furthermore, since the hydrated layer of OCP is almost certainly hydrophilic in nature, it is more likely than the apatitic layer to form a low energy interface with aqueous phases. For HAP, $\gamma_{s}^{-}=16.0 \mathrm{mJm}^{-2}$ while for OCP $\gamma_{s}^{-}=19.7 \mathrm{mJm}^{-2}$.

The flocculation and repeptization of hydroxyapatite may be important in the formation and destruction of calcified tissues and may contribute to their structural strength and hardness. Flocculation may also play a role in pathological conditions such as ectopic calcification, dental calculus and urinary stones. In a dispersion study, three suspensions of calcium phosphates (DCPD, OCP and HAP) were prepared in $0.1 \mathrm{~mol} \mathrm{~L}^{-1} \mathrm{NaCl}$ solutions. It was found that the OCP and HAP suspensions collapsed immediately while DCPD remained stable for more than two hours ${ }^{14}$. The fact that the dispersion behavior of these calcium phosphates could be explained by considering only the Lewis acid-base interaction is an indication that in polar and especially in aqueous solutions Lewis acid-base energies, whether repulsive or attractive, commonly are much more important than Lifshitz-van der Waals and electrostatic interactions at smaller separations. The critical parameter that accounts for the different behavior of DCPD was also $\gamma_{s}^{-}$, the electron donating parameter of the surface (Table 1). The hydrophilicity of the DCPD surface may result from its crystal structure which consists of chains of $\mathrm{CaPO}_{4}$ arranged parallel to each other with lattice water molecules interlayered between them. The higher value of $\gamma_{s}^{-}$for DCPD was 
responsible for the negative interfacial tension against water. This net negative interfacial tension normally is associated with a thermodynamically unstable interface. Such a system will tend to spontaneously relax by either abolishing the interface or by reverting to a more stable geometric configuration, ultimately resulting in zero interfacial free energy. The strongly asymmetric Lewis acid-base forces (usually high $\gamma_{s}^{-}$) are probably responsible for the orientation of water molecules adsorbed on the surfaces. Water molecules oriented on the surface of one particle will repel those oriented in the same manner on the surface of adjacent particle. If the orientation of the water molecules by surface of the particles is sufficiently strong, the two particles will not be able to approach each other.

\section{ACKNOWLEDGMENT}

We thank the National Institute of Health (Grant DE 03223) for support of this work

\section{REFERENCES:}

1. Wu, W. and Nancollas, G.H. Adv. Colloid Interface Sci., 79, 229 (1999).

2 W. Stumm and J. J. Morgan, Aquatic Chemistry, Wiley Interscience, New York, 1996, pp. 516-816.

3. M. L. Dundon and E. Mark, J.Am. Chem. Soc., 45, 2479 (1923).

4. Von W. J. Jones, Z. Phys. Chem., 82, 448 (1914).

5. V. B. Ratinov and O. M. Todes, Dokl. Akad. Nauk. SSSR, 132, 402 (1960).

6. A. E. Nielsen, Kinetics of Precipitation, The Macmillan Company, New York, 1964.

7. C. J. van Oss, Interfacial forces in aqueous media, Marcel Dekker, New York, 1994.

8. S. Mann, B. R. Heywood, S. Rajam and J. D. Birchall, Proc. R. Soc. London, Ser. A 423, 457 (1989).

9. B. R. Heywood and S. Mann, Langmuir, 8, 1492 (1992).

10. J. O. Titiloye, S. C. Parker, D. J. Osguthorpe and S. Mann, J. Chem. Soc. Chem. Commun., 1494 (1991).

11. I. Weissbuch, L. Addadi, M. Lahav and L. Leiserowitz, Science, 253, 637 (1991).

12. L. Addadi, I. Weissbuch L, J. Vanmil, L. J. W. Shimon, L. Leiserowitz, Z. Berkovitchyellin and M. Lahav, Angew. Chem. Int. Ed. Engl. 24, 466 (1985).

13. C. J. van Oss and R. F. Giese, Clays and Clay Minerals, 43, 474 (1995).

14. Y. Liu and G. H. Nancollas, J. Phys. Chem., 101, 3464 (1997) 